\title{
Intracerebellar haematoma
}

\author{
HUGH ARONSON, SHERIF SHAFEY ${ }^{1}$, AND FREDIE GARGANO \\ From the Departments of Neurosurgery, Neurology and Radiology, University of Miami School of Medicine, \\ Miami, Florida, U.S.A.
}

Until the report of McKissock in 1960, no significant series of surgically treated cases of spontaneous intracerebellar haemorrhage had been recorded (McKissock, Richardson, and Walsh, 1960). Earlier reports had dealt mostly with post-mortem studies (Hyland and Levy, 1954). It is generally conceded that in the neighbourhood of $10 \%$ of spontaneous haemorrhages into the brain will occur in the cerebellum (Mitchell and Angrist, 1942). This may be related to the fact that the cerebellum represents approximately $10 \%$ of total brain weight. A patient, recently admitted to the Jackson Memorial Hospital, illustrated most of the important clinical facets of this condition. Appropriate diagnostic studies enabled us to make the correct diagnosis preoperatively.

\section{CASE HISTORY}

M.S., a 57-year-old Negro, arrived at the Emergency Room of the Jackson Memorial Hospital on 23 October 1963, complaining of severe occipital headache of several hours' duration. The headaches, beginning in the morning, gradually increased in severity and developed into 'the severest headaches I have ever had in my life'. During the same interval, the patient vomited four times. He was conscious and had no seizures, sensory or motor abnormalities.

The patient had been hypertensive and had suffered from headaches for 15 years. Recently, he had experienced vague episodes of dizziness.

Physical examination on admission revealed a conscious, somnolent, cooperative man in severe distress. Blood pressure was $220 / 110 \mathrm{~mm} . \mathrm{Hg}$; pulse 70 and regular; respirations 16 and regular; temperature $98^{\circ} \mathrm{F}$. Except for the hypertension, general medical examination was within normal limits.

On neurological examination, the cranial nerve signs were most revealing. Visual acuity was grossly intact, as were the visual fields. Fundoscopic examination revealed grade II hypertensive retinopathy bilaterally. The pupils were $4 \mathrm{~mm}$., round, regular and equal. They reacted to light directly and consensually. Nystagmus was present with the eyes in the primary position, but most marked on right lateral gaze. Horizontal, vertical, and rotary components were seen. At times, dissociated nystagmus was seen. There was limitation of conjugate gaze to the right on voluntary movements, following 'Present address: Department of Neurology, Jackson Memorial Hospital, Miami, Florida. movement, and both Doll's head manoeuvre and caloric stimulation. Optico-kinetic responses were impaired in all directions, most severely in the vertical direction. A right lower facial weakness was noted. No other cranial nerve signs were seen. Tone, power, and coordination of the extremities were within normal limits. Deep tendon reflexes were symmetrically active. There were no pathological reflexes. Sensory testing was normal. Specific tests of cerebellar function were normal. There was marked nuchal rigidity. The clinical impression was of a ruptured aneurysm in the posterior fossa.

The day following admission, the patient's headache was more severe and he complained of vertigo and diplopia. The paresis of right conjugate gaze was more apparent. Forty-eight hours after admission the patiene became agitated and confused. Paralysis of right conju尺 gate gaze was noted and dissociated nystagmus was marked. The pupils became myotic, $2 \mathrm{~mm}$.; respiration became Cheyne-Stokes in type; and a right hemiparesis became apparent for the first time.

Skull radiographs were unremarkable.

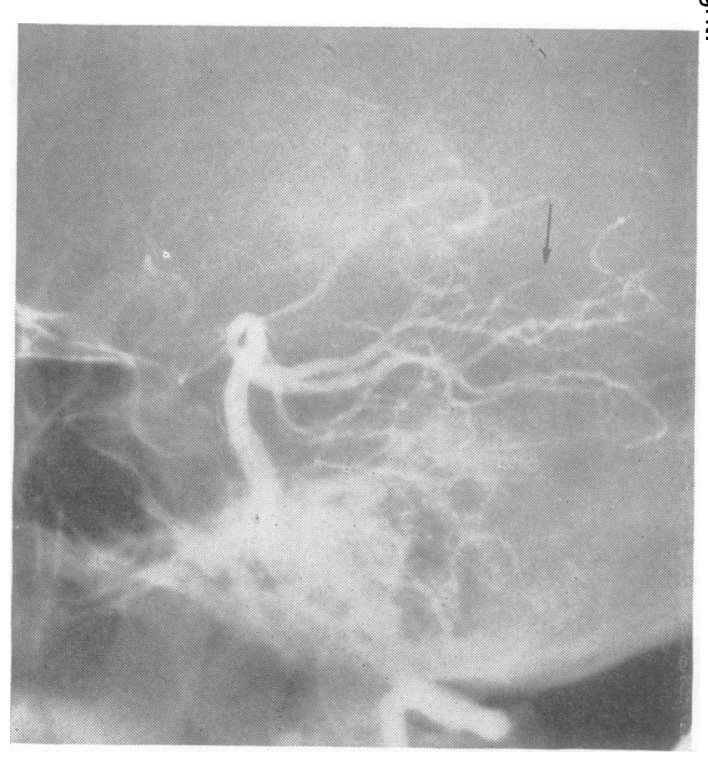

FIG. 1. Left vertebral arteriogram shows upward displacement of medial branches of superior cerebellar arteries, indicating ascending cerebellar herniation. 


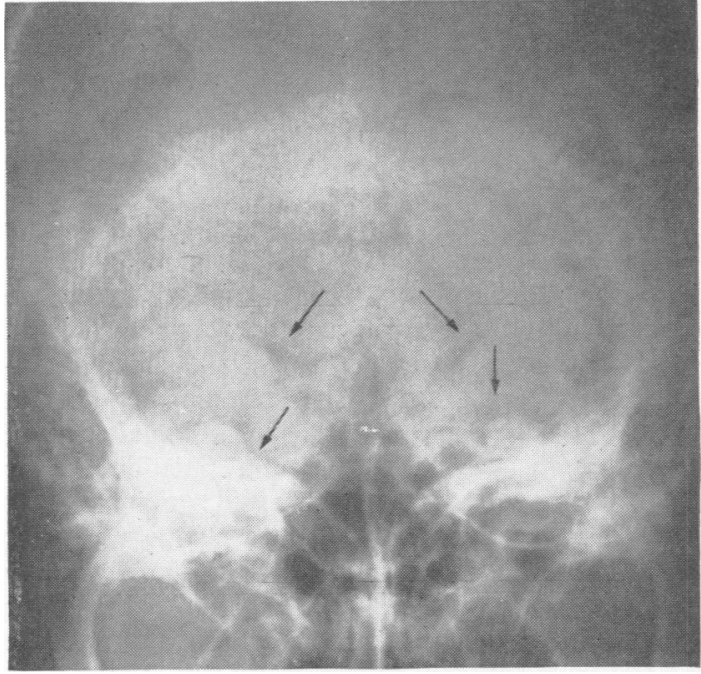

FIG. $2 \mathrm{a}$

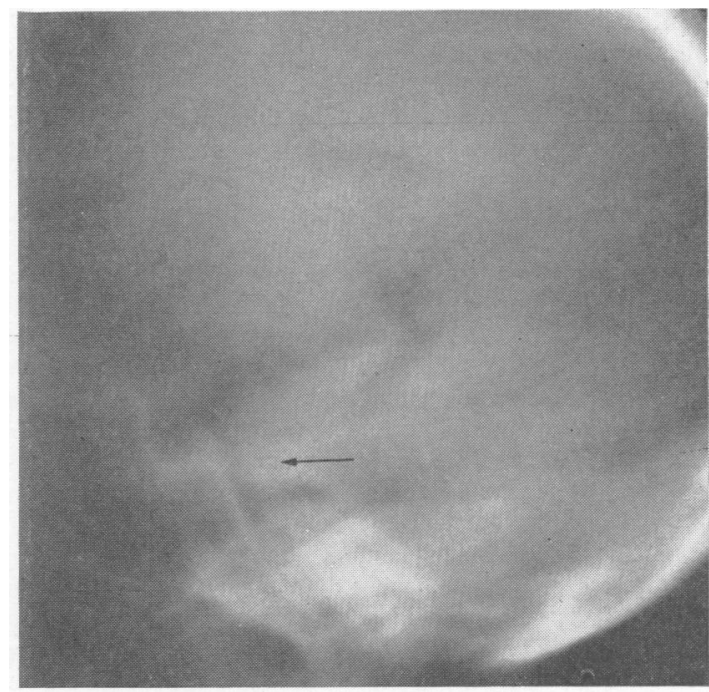

FIG. $2 b$

FIGS. $2 \mathrm{a}$ and $2 \mathrm{~b}$. Small pneumoencephalogram without fluid exchange shows non-filling of ventricular system, poor filling of the cisterna magna, narrowing of the cerebello-pontine angle cisterna, anterior tilting of the quadrigeminal plate cisterna, and asymmetry of the crural cisterns. The right cerebello-pontine angle cistern is narrower than the left.
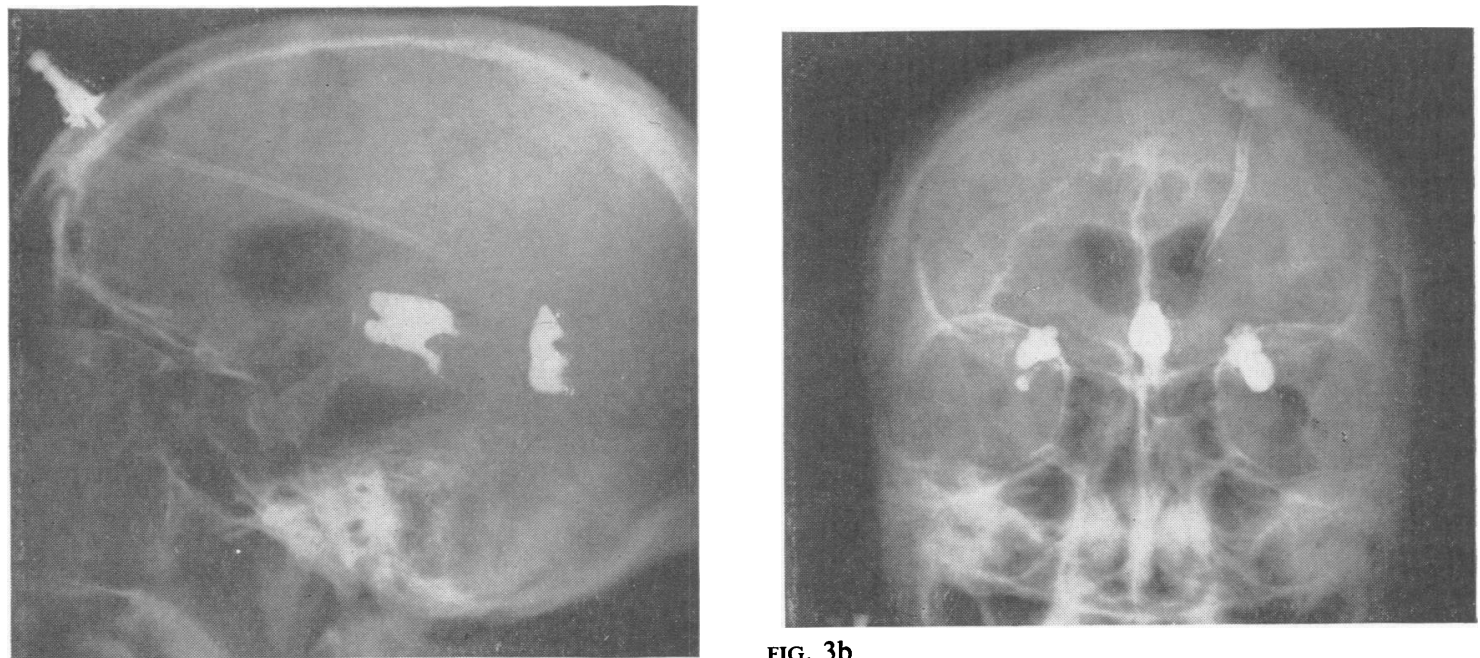

FIG. 3a

FIG. $3 b$

FIGS. 3a and 3b. Positive contrast ventriculogram shows kinked aqueduct which was blocked at its junction with the fourth ventricle. There is no displacement of the fourth ventricle from the midline.

The initial contrast study was a left vertebral arteriogram (Fig. 1). This demonstrated upward displacement of the medial branches of the superior cerebellar arteries, indicating ascending cerebellar herniation.

A small pneumoencephalogram, without fluid exchange, was performed (Fig. 2 a, b). There was nonfilling of the ventricular system. Examination of the cis- terns showed poor filling of the cisterna magna, narrowing of the pontine angle cisterns, anterior tilting of the quadrigeminal plate cistern, and asymmetry of the crural cistern. The right cerebello-pontine angle cistern was narrower than the left. A diagnosis of right cerebellar hemisphere haematoma was made.

To corroborate the diagnosis, a positive contrast 
ventriculogram was done (Fig. 3, a, b). This study showed a kinked aqueduct which was blocked at its junction with the fourth ventricle. There was no displacement of the aqueduct from the midline.

Because of the progressive nature of the patient's lesion, he was taken to the operating room. Under general anaesthesia and in the sitting position, the posterior fossa was opened through a midline incision. A small amount of subdural fluid was found surrounding the right cerebellar hemisphere. On needling the right hemisphere, a solid clot of blood, $2 \times 2 \mathrm{~cm}$., was encountered, $1 \mathrm{~cm}$. from the surface of the cerebellum. Through a transcerebellar incision, the entire haematoma was removed. Spinal fluid with Pantopaque could then be seen running from the outlet tracts of the fourth ventricle. Immediately after removal of the haematoma, the patient's vital signs improved and his respirations became regular.

The day following surgery, the patient was more responsive, respirations were regular, and vital signs were stable. Thirty-six hours after surgery, the patient suddenly developed bleeding from the wound edges, and a previously placed tracheotomy wound. In spite of vigorous therapy, including vitamin $\mathrm{K}$ and fresh plasma, the bleeding could not be stopped. A ventricular puncture was performed and produced clear fluid under normal pressure. In spite of vigorous medical treatment, the patient succumbed to the bleeding episode which could not be explained in spite of a multitude of haematological studies.

Post-mortem examination was refused by the family.

\section{DISCUSSION}

Posterior fossa haematomas tend to present a typical clinical picture (Hyland and Levy, 1954; Gross, 1955; McKissock et al., 1960; Dimsdale, 1964). First, they demonstrate signs common to intracranial bleeding in any location, such as headache, vomiting, and mental confusion. In addition, however, the clinical triad of myosis, conjugate gaze palsy, and irregular respirations, have been found with great frequency in intracerebellar haematomas.
Both in Hyland's and McKissock's series (1954, 1960) this triad was frequently encountered. It is significant that a minority of patients have either symptoms or signs directly referrable to cerebellar dysfunction.

The underlying pathology has revealed hypertension to be the single most important cause (Hyland and Levy, 1954). A branch of the anterior superior cerebellar artery which supplies the dentate nucleus is the most frequently ruptured vessel. One-fifth to one-fourth of the patients have angiomatous malformations. The remainder have a variety of underlying conditions, including aneurysm, blood dyscrasias, meningitis, and poisoning. Our patient demonstrates a hypertensive aetiology.

Previous reports have failed to evaluate various $x$-ray studies. Carotid and vertebral angiography produced subtle but definite changes. A cisternal air study, combined with ventriculography, was of the greatest help in anatomically defining the lesion. Surgical mortality in this condition is high, being $36 \%$ in McKissock's series. Extension of the haematoma into the brain-stem, post-operative oedema, and irreversible brain-stem injury have con-o tributed to the high mortality figures. Perhaps o $\vec{\Phi}$ greater significance, however, is the fact that two@ thirds of these patients who survive long enough fog diagnostic studies to be carried out can be saved with prompt surgical intervention.

\section{REFERENCES}

Dimsdale, H. B. (1964). Spontaneous hemorrhage in the posterior fossa. Arch. Neurol. (Chic.), 10, 200-217.

Gross, S. W. (1955). Posterior fossa hematomas. J. Mt Sinai Hosp. 22, 286-289.

Hyland, H. H. and Levy, D. (1954). Spontaneous cerebellar haemorrhage. Canad. med. Ass. J., 71, 315-323.

McKissock, W., Richardson, A., and Walsh, L. (1960). Spontaneous cerebellar haemorrhage: a study of 34 consecutive cases treated surgically. Brain, 83, 1-9.

Mitchell, N., and Angrist, A. (1942). Spontaneous cerebellar hemorrhage. Amer. J. Path., 18, 935-953. 